\title{
Survey Paper on Wireless Underground Positioning System
}

\author{
Sapna Kataria \\ M.Tech-CSE \\ NCU University \\ Gurgaon, India
}

\author{
Poonam Singh \\ M.Tech-CSE \\ NCU University \\ Gurgaon, India
}

\author{
Prachi Ahlawat \\ Associate Professor \\ NCU University \\ Gurgaon, India
}

\begin{abstract}
This paper discuss the structural design of staff positioning techniques in unsafe places in underground mines, this paper also highlights the gains and weaknesses of wireless sensor network technology and address an economical, continuous monitoring system of covert mine workers security. It can realize the effective management of precise positioning of man and machine. This article presents the composition of system, the enactment and management of network formation. The actual application shows that the ZigBee technology can play an important role in underground personnel information and security management.
\end{abstract}

\section{General Terms}

Staff positioning in underground mines

\section{Keywords}

ZigBee, RFID, GPS technology, wireless network.

\section{INTRODUCTION}

Mining industry is the chief industry of our country, Miners work underground under the earth, so positioning and monitoring are important for safe production ${ }^{[1]}$. These industrial sites have a lot of hazardous areas and due to environmental situations; sometimes it is challenging for personnel monitoring system to preserve security. Negligence in the safety may cause harm to high value equipment or loss of human existence. Now because the underground underpass are crisscross and the staff working area is widespread so to get underground personnel real-time dynamic information the real time locating management is particularly important. Communication is the most dynamic key factor, to monitor different constraints constantly and to take necessary actions accordingly, To enhance security, safety and efficiency in underground mines, a reliable communication system must be established between workers, moving in the mine, and a fixed base station or control center on the earth. The communication network must not be disturbed at any moment and at any state. The wired communication network system is not so effective, because the installation charge as well as maintenance charge is high, it is very difficult to re-install the wired communication system after destruction due to any reason. The damage may cause temporary interruption or may cause a long duration break down of the system.

Wireless communication is the actual idea for mine safety monitoring; many diverse research ideas are projected on wireless communication. Wireless positioning subsystem is the elementary idea of the system. There are numerous operational positioning and navigation technologies like GPS (global positioning system) and the positioning technologies based on ZigBee or RFID, CWUMSN (chain-type wireless underground mine sensor network), and so on. it is a 2 -way wireless communications technology which have diminutive distance, little complexity, small power use, less data rate, comparatively low-cost, great reliability, modest networking and flexibility ${ }^{[5]}$. This positioning system can return exact staff changing aspects to the intelligent monitoring system on earth and command center.

\section{RELATED WORK}

Recently underground mining technology communication is the obvious need, for this several techniques are applied many research papers have been written, some of them are discussed here. In 2008, Hauli Chen and Zhifen Feng has done research on design of data acquisition and record system based on ZigBee and GPS. After that in 2011, Zhiyong Cao, Hanyu Lu and Quimin has done research on underground staff positioning system based on wireless sensor network technology. In 2013, Li Zhi and team members worked on the research staff position system of wireless sensor networks in dangerous places, and so on. After so much research work already done;

In this paper, several underground navigation technologies which are possible are studied and investigated and find out in which situation which technology is best suited. section 1 , is introduction part, then we have described the related work in section 2,after that section 3 consist of description of various technologies, section 3.1 discuss about GPS technology \&sec. 3.1.1 consist its advantages and disadvantages, in section3.2 we discussed about RFID technology and then about ZigBee in section 3.3, section 4 consist of conclusion of the survey, and section 5 has acknowledgement part,and the last one is the refrences.

\section{Technologies Description}

\subsection{GPS Technology}

Gps technology is most frequently used. The satellites send the signals, after that the distance is estimated between the receiver and satellites with time variance, the triangulation process are used to estimate a client's location ${ }^{[7]}$. GPS works truthfully in all climates, day and night, and around the world. No payment needed for using GPS signals. GPS signals could be jammed by walls, dense forest, and multistory building. They don't enter indoor spaces well; therefore some places may not document exact GPS navigation technology. 


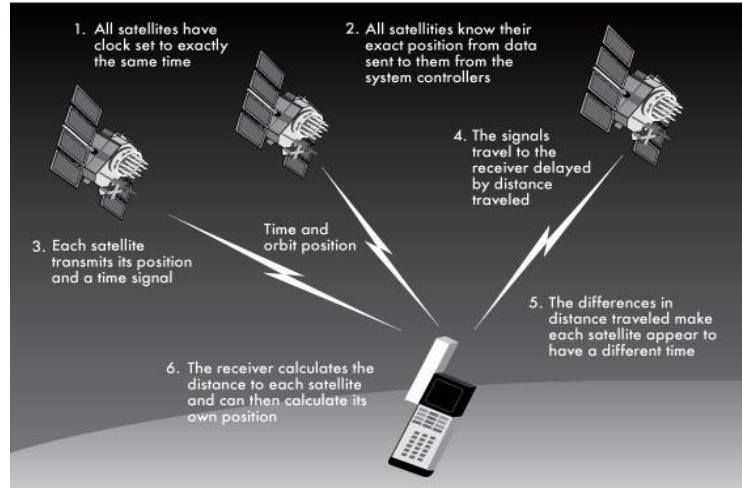

Figure 1-Gps working diagram

GPS satellites revolve around our planet (earth) twice a day in a specific orbit and transfer signal information to ground. GPS receivers use these signals and the triangulation method to compute the user's precise location. Basically, the GPS receiver equates time, a signal was transferred by a satellite with the period it was acknowledged. The time variance tells the receiver how far the satellite is. Now, the distance measures from a few other satellites, with this the receiver can conclude the user's location and presents it on the electronic map. The receiver should be locked on to the signal of at least three satellites to calculate a two dimensional locus and with four or more satellites in sight; the receiver can govern the user's three dimensional location. Once the user's location has been calculated, the GPS unit can analyze other information, like speed, tour distance, sunrise and sunset time and distance to destination, etc. signals from only three satellites are necessary to carry-out trilateration procedure; determining of your position on ground is based on your distance from the three satellites. The signals from the fourth satellite are fired and are used to approve the results of the preliminary calculation. If the position determined from distances of satellites "A-B-C" don't match with the calculation based on "A-B-D" then other arrangements are tried until a reliable result is achieved.

\subsubsection{Advantages and disadvantages of GPS}

GPS is enormously stress-free to navigate as it tells you the route for each turn you should take or you have to; to reach to your last stop. GPS works in all-season so you don't need to fear about the environmental conditions as in other directing devices. GPS is cost effective in contrast with the other map reading systems. The most striking quality of GPS system is its $100 \%$ coverage on the globe. It also aids us in hunting the nearby restaurants, gas stations, petrol pumps, bank branches etc. and it is very valuable for a new place. Because of its less cost, it is very easy to assimilate in another technology for e.g. mobile phone. The GPS system is frequently updated by the US government and therefore it is very advance. It is the finest directing system in water also; as in larger water bodies we are often deluded due to absence of proper instructions. Sometimes it may be unsuccessful due to certain reasons and for that situation you should bring a backup map. If you are using a battery functioned device for GPS, there could be a battery letdown and you may need some outside power supply which is not always conceivable. Sometimes these signals are not precise due to some hindrances to the signals like trees, buildings, and climatic conditions for e.g. geomagnetic rainstorms.

\subsection{RFID Technology}

RFID is a non-contact and two-way communication technology, which can bring about the automatic ID and data exchange to people and equipment. The positioning and navigation system based on RFID system has been encoded the data transported by wireless through the random sequence, it also has a fairly perfect communication protocol with the intimate anti-collision mechanism so that it can identify multiple fast-moving targets concurrently ${ }^{[2-4]}$. Generally this system can work in the $433 \mathrm{MHz}$ band. While sources of the disruption are more than it works in the $2.4 \mathrm{GHz}$ band, the communication distance will be even better, which will be up to $100 \mathrm{~m}$. The choice of frequency will affect communication speed and position error. The positioning could be within 10$30 \mathrm{~m}$ and transmission speed at $64 \mathrm{kbps}$ when it works in the $433 \mathrm{MHz}$ band, which also fulfills the necessity in the absence of voice and video transmission. However most of the base station launching capacity is over $15 \mathrm{~W}$ and the price of it is higher than other types of sources.

\subsubsection{The working principle of the positioning} and navigation system based on RFID

Positioning and navigation system founded on RFID is mainly built with control center and the underground part. Control center is contains server, printers, monitors and other components. Control center processes the information returned from the underground stations. Underground portion comprises of base stations (card reader) and mobile navigating instruments. Base station not only can launch the wireless signal to activate the ID card and receive the message from the ID card, it also can display itself 3-D location because it was added to monitor module. Mobile navigating instrument as well as the ID card module and has been added the navigation system module. Navigation system module is made up by regularly updated three-D electronic map of the underground mine and has the basic navigation roles. The card reader intermittently launches RF (Radio frequency) signal to intellects the ID module, then it will couple with ID card and collect ID card information (card numbers and miners information). The card reader guides this message to the control center over fiber-optic. So the control center can determine the precise information (location information, basic information) of the miner after processing the information, and the dynamic information of the underground miner can be organized in real-time by the control center.

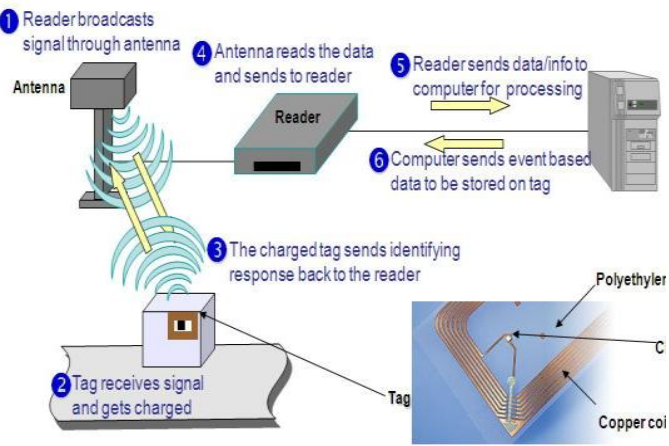

Figure 2-RFID Operation

\subsubsection{Advantages and disadvantages of RFID Technology}

In the RFID technology there is no need that the tags should to be in line of eyesight with the receiver to be read. These tags can store a lot of statistics, and could follow commands. They Has the capability to determine the position. This is a 
flexible technology: it can be reduced to thumb tack or can be of a tablet size, dependent on its use. According to a report, it was determined that the can RFID actually enriched each supply chain's system presentation and augmented projected profit. But on the other hand Active RFID could be costly due to the use of batteries, it still needs to regulate RFID strategies, with RFID devices there is a privacy concern. RFID could be easily interrupted, even if it is encoded. We have to invest a long time to build RFID device's suits Because of geographical complexity and bad environment wired connection has problems in data transfer. RFID technology also has personnel positioning inaccuracy problem.

\subsection{ZigBee technology}

$\mathrm{ZigBee}$ is a wireless communication protocol. The ZigBee architecture is made by the application layer, network layer, application support layer, data link layer, the media access layer and physical layer. ZigBee is a communication technology with low power, low complication and low cost, bidirectional. The range of the system is from 10 meters to 200 meters, and the card readers' speed is $20 \mathrm{~m} / \mathrm{s}$ or more. This system uses the $2.4 \mathrm{GHz}$ band and the strong DSSS (Direct Sequence Spread Spectrum) communication technique, the transmission rate is $20 \mathrm{kbps}$ to $250 \mathrm{kbps}$ (which can meet the needs of voice communications). This system has low cost base stations, its network capacity is so larger that it can accommodate up to 65,500 (approx $)^{[6]}$ devices.

In ZigBee technology identity cards are used so the accurate work of mine workers could be calculated every day, their time of coming in and going out of the mine. Moreover, supervisory department could also monitor remotely with the help of Internet. When the monitoring system is not working normally, The system automatically controls the personnel location and after computing the nearest safe place, It guides the persons to the safe ground before the complete disaster of communication systems. The ZigBee system usually separated into two portions: ground monitor system and underground equipment. On the basis of genuine application environment, the system based on Zigbee has a control center on the ground, base stations and navigators are underground.

Control center: The control center is accountable for the storage calculation and examination of the information transmitted throughout the network. The control center has three-dimensional electronic map of the underground mine.

Base station (card reader): Underground base station's main function is to collect information from nodes and to judge the location of the miners. In addition, the base stations also provide voice information transmission.

Navigator: Navigators carried by miners are built up of two parts, one is the position node and the other is speech communication.

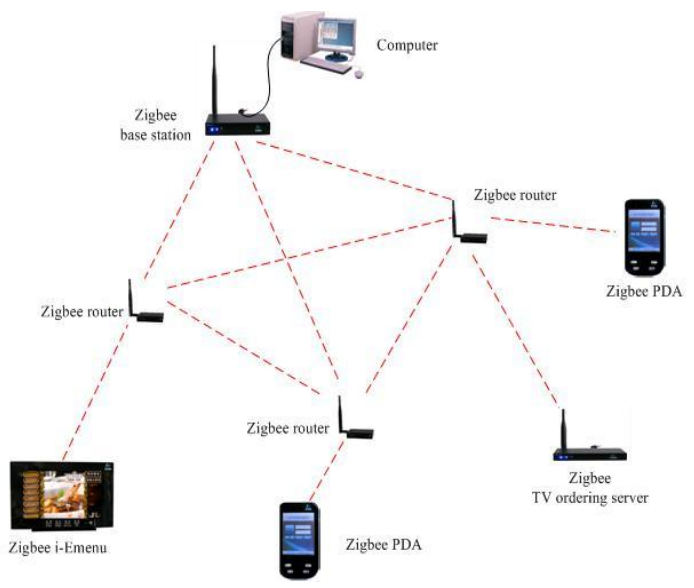

Figure 3-ZigBee Technology based Model

Mine workers carries Micro-communications module fixed ZigBee chip, which are power-driven by 2 AA batteries, also known as the mobile node ${ }^{[6]}$. Positioning nodes launch the signals which were received by the base station. Base station governs the location of the positioning node (According to the signal strength) and transmits the location to the control center. Control center examine the terminal location information and underground circumstances and then directs to the terminals in the electronic map and passes the message through the voice communications ${ }^{[6-7]}$.Terminals also can demand control center for the navigation by voice communications when required. According to this principle the navigation system can reach real-time navigation. If during transmission any data packet fails to send, then the system will itself resend those packets. Its network structure is distinctly appropriate, like mesh, star, cluster-tree, adhoc and P2P topology in Network layer. For altering the network topology into other practices, Network layer permits routing and multihopping. Encoded security keys are established and swapped in MAC, Application and network layer for trustworthy communication. In couple of years ZigBee will be used in the area of Industry control, Industry wireless location, Home network, Building mechanization, Medical apparatus control, Mine safety, Analyze and calculate electricity consumption of users, Home automation or smart home etc.

\subsubsection{Problems with ZigBee}

ZigBee is a advanced and effective technology but still there are some areas that needs improvement. The coordinator brings too much nodes, particularly in the large scale wireless network. The nodes carries batteries, there are some places where it is difficult for humans to change the batteries of nodes. And a large number of nodes cause problems in changing presents and enhanced design. the coordinator only deal with the charge on the Zigbee network, the rest tasks will be processed by alternative processor.

Table 1 Comparison of ZigBee, RFID \& GPS Technologies

\begin{tabular}{|l|l|l|l|l|}
\hline $\begin{array}{l}\text { S. } \\
\text { No. }\end{array}$ & characteristics & ZigBee & RFID & GPS \\
\hline 1. & Battery life (days) & $100-1000+$ & $\begin{array}{l}\text { No battery } \\
\text { (passive tags) }\end{array}$ & $1-7$ \\
\hline 2. & Range (meters) & $1-75 \mathrm{~m}$ & $1 \mathrm{~m}$ & $1000+$ \\
\hline 3. & Power consumption & Low & Low & high \\
\hline 4. & Data transfer rate & low & high & low \\
\hline
\end{tabular}




\begin{tabular}{|l|l|l|l|l|}
\hline 5. & Nodes per network & $255-65 \mathrm{k}+$ & $650+$ & 1000 \\
\hline 6. & Key attributes & $\begin{array}{l}\text { Reliable, } \\
\text { cost } \\
\text { effective }\end{array}$ & $\begin{array}{l}\text { No human } \\
\text { interference, } \\
\text { cost efficient. }\end{array}$ & $\begin{array}{l}\text { Reach } \\
\text { qualit } \\
\text { y, } \\
\text { min. } \\
\text { cost } \\
\text { compa } \\
\text { rativel } \\
\text { y. }\end{array}$ \\
\hline 7. & distance & medium & short & long \\
\hline 8. & Cost & High & Medium & low \\
\hline
\end{tabular}

\section{CONCLUSION}

By applying the innovative wireless communication technology in man and machine positioning system of secretive coal mines, it is studied that GPS as a navigation system, it cannot enter into the ground because of the low signal strength, so it cannot be realistic to underground mine. On the other hand, the native coal mine personnel positioning system generally using RFID technology; the foremost purpose of the system is staff attendance, but the accuracy is not enough. ZigBee wireless location technology is a major highlight of the mine to improve safety, protect life and property of the people. it can prevent the ultra-capacity production and prevents personnel to enter hazardous areas. Meanwhile, it also provides strong technical support and IT assurance to digitized mine construction. Underground manmachine positioning and information supervision is not only important for effective rescue work, but also it is important to improve the level of present-day management of mine enterprises

\section{ACKNOWLEDGMENTS}

Sincere thanks of gratitude to the expert Mrs. Prachi Ahlawat, assistant professor at NCU university, Gurgaon, India, who has given her precious time and support, and highly grateful to her invaluable constructive criticism and friendly advice, for sharing her truthful and illuminating views on a no. of related issues. She contributed towards development of the template.

\section{REFERENCES}

[1] A staff position and mine environmental monitoring system. ZHAO Guangyuan, ZHAGN Di ,LI Weihua. Safety in Coal Mines 2009.

[2] The coal mine staff based on RFID Location System. MINXiaoyong. Mining \& Processing Equipment,2007.

[3] RFID-based positioning system of underground personnel. LUO Xiandong, TANG Chong, Zhao Weihua Mining Technology 2007.

[4] RFID technology-based positioning system of coal mine workers. ZHUAGN Kun, Sun Zhe. Computer Applications..2007.

[5] GIS based on ZigBee and Underground staff Positioning System. Li Wei,CUI Jian ming. Industry and Mine Automation, 2010.

[6] Underground staff Positioning System Based on Wireless sensor network, Zhiyong Cao, Hanyu Lu, Qimin He. 2011.

[7] "The global positioning system", IEEE Transactions on Microwave Theory and Techniques, McNeff, J.G., Vol. 50, pp. 645-652, Mar. 2002.

[8] https://www.google.co.in/search?q=rfid+operation\&biw $=1366 \&$ bih $=663 \&$ source $=\operatorname{lnms} \&$ tbm $=i s c h \& s a=X \& v e d=$ 0CAYQ_AUoAWoVChMIo9K6wdXQyAIVDhuOCh2s XAtu\#imgrc=asKod8J4rEStiM\%3A.

[9] https://www.google.co.in/search?q=zigbee\&es_sm=93\& source $=$ lnms \& tbm $=$ isch \&sa $=X \&$ ved $=0 C A c Q \_A U o A W o$ VChMI7LOe0dbQyAIVRASOCh2thghY\&biw=1366\&b $\mathrm{ih}=663 \#$ tbm=isch\&q=2010101146346798. 